\title{
The Influence of Physical Function on Drawing Performance and Drawing Motion in Clumsy Children
}

\author{
Chisa Ikeda ${ }^{1}$, Sonomi Nakajima ${ }^{2}$, Toshio Ohyanagi ${ }^{3}$, Yuji Nakamura ${ }^{2}$, \\ Yasuhito Sengoku ${ }^{2}$ \\ ${ }^{1}$ Special Needs Education Course, Hokkaido University of Education, Japan \\ ${ }^{2}$ Department of Occupational Therapy, School of Health Sciences, Sapporo Medical University, Japan \\ ${ }^{3}$ Department of Liberal Arts and Sciences, Center for Medical Education, Sapporo Medical University, Japan
}

\begin{abstract}
Background: Children with developmental disorders who require occupational therapy often show clumsiness in handwriting (hereafter referred to as clumsy children). The quality of handwriting has been reported to be deteriorated in children who had physical function-related issues for using handwriting tools. However, to date there has not been any clinical research to investigate the types and severity of deficits in physical function that influence the using handwriting tools. Similarly, how the trends and the severity of problems in physical function affect the characteristics of the results of handwriting task was previously unaddressed.

Objective: This study investigated the influence of characteristics of physical function on drawing performance and drawing motion in clumsy children.

Method: Participants were healthy children and clumsy children enrolled in grades 2-6 of elementary school. The participants' drawing performance was evaluated using the triangle drawing task on a tablet personal computer. The participants' drawing motion was evaluated using the two-dimensional movement analysis device. The physical function of clumsy children was evaluated using the clinical observational assessment.

Results: The largest stray distance, the range of speed and the difference of speed for the healthy child group by grade and each clumsy child. The sway length ratio of the clumsy children differed from those of the healthy children group. Clumsy children who exhibited poor drawing skills also had problems in posture maintenance, muscle condition, upper limb coordination, and eye movement.

Conclusion: We found the influence of poor physical function on poor drawing and drawing motion in clumsy children.
\end{abstract}

Keywords: developmental disabilities, clumsy children, drawing performance, drawing motion, physical function

(Asian J Occup Ther 15: 37-44, 2019)

\section{Introduction}

Children with developmental disorders who require occupational therapy often show clumsiness in writing and drawing (hereafter referred to as clumsy children) [1].

Previous studies have examined the accuracy of

Received: 26 February 2018, Accepted: 29 January 2019

Corresponding to: Chisa Ikeda, Special Needs Education Course, Hokkaido University of Education, 5-3, Ainosato, Kita-ku, Sapporo, Hokkaido, 002-8501, Japan

e-mail: nikonikochisa57@gmail.com

C2019 Japanese Association of Occupational Therapists drawing tasks using PC tablets to measure changes in speed and pen pressure while drawing figures. These studies have reported that clumsy children exhibit a lower level of accuracy in drawing and show patterns of drawing speed changes that are different from those of children with typical development (hereafter referred to as healthy children) [2-4]. These studies also noted that accuracy and speed changes both differ greatly between individual clumsy children. However, clumsy children who present with problems associated with using a pen are uniformly assessed as having poor handwriting skills, then clinical support based on individual problems has not been examined.

Previous reports have identified numerous factors 
that affect the test results of children assessed as having poor handwriting skills in using a pen. These factors include problems in physical function, such as decreased cooperativeness and manual dexterity of the upper limbs, difficulty in maintaining posture, controlling muscle tone and poor eye movements. Additionally, the quality of handwriting has been reported to be deteriorated in children who had physical function related issues for using handwriting tools [5-9]. However, to date there has not been any clinical research to investigate the types and the severity of deficits in physical function that influence the using of handwriting tools. Similarly, how the trends and the severity of problems in physical function affect the characteristics of the results of handwriting task was previously unaddressed.

In this study, we clarified the relationship between drawing performance and drawing motion in clumsy children as compared to that in healthy children by analyzing the results of a drawing task and their drawing motions while doing the task. Additionally, by analyzing physical function we also elucidated how characteristics of physical function influence drawing performance and drawing motion in clumsy children.

The hypotheses of this study were as follows.

1) The clumsy children were different from the healthy children in the accomplishment result of drawing motion in the drawing task.

2) The accomplishment result and the campaign for drawing motion in the drawing task of the clumsy children were related to characteristic of physical function.

\section{Methods}

\section{Research Design}

A cross-sectional design was used to study the relationship between drawing performance and drawing motion in clumsy children, and to determine how characteristics of physical function influence drawing performance and drawing motion in clumsy children. Each clumsy and healthy child was asked to perform a drawing task, and each clumsy child was asked to perform an additional physical function test. This study was approved by the institutional review board at Sapporo Medical University. Parents were informed of the study and given a right to remove their child from this study.

\section{Subjects}

The clumsy child group comprised 10 boys who had received a diagnosis of developmental disorders by a doctor, and who showed difficulty in handwriting. There is not an evaluation scale of clumsiness standardized in Japan. Therefore, in this study, the clumsy child was defined as judged to be weak in writing compared to each grade children by occupational therapists and parents. This group included 2 second grade students, 3 third grade students, 1 fourth grade student, 2 fifth grade students, and 2 sixth grade students.

The healthy child group comprised 54 boys who did not have any apparent physical or learning disabilities. We classify them in 2-6 grade by age. The healthy child group included 8 second grade students ( $7-8$ years old), 10 third grade students (8-9 years old), 12 fourth grade students (9-10 years old), 12 fifth grade students (10-11 years old), and 12 sixth grade students (11-12 years old),. All subjects normally used their right hand to handwrite.

\section{Instrument}

\section{Drawing Performance and Drawing Motion}

A tablet personal computer (Sony VAIO Duo 11; hereafter referred to as PC tablet), a stylus to draw directly on the screen, and software developed for the assessment of upper limb function in handwriting tasks [10] were used for the drawing task. Microsoft Visual Basic 2012 was used to create the figures used for assessing upper limb function. During the drawing task, changes in the positioning of the stylus and elapsed time were recorded using a sampling frequency of $133 \mathrm{~Hz}$.

Subjects were seated with both feet placed on the floor and with their forearms on the table. The PC tablet was placed $12.5 \mathrm{~cm}$ from the front edge of the desk, and subjects held the stylus with their right hand. Their left hand was placed on the bottom left of the tablet. Video cameras were placed above and to the right of the subjects to record the drawing motion. Based on a previous study [1], markers were placed on the right acromion, lateral epicondyle of the right humerus, right radial styloid process, and stylus to serve as indicators for the analysis of motions.

The drawing task was to draw a line between two concentric equilateral triangles displayed on the PC tablet, spaced $3 \mathrm{~mm}$ apart, the side length of the outer equilateral triangle was $10 \mathrm{~cm}$. The subjects drew a line in a counterclockwise direction, starting from the top point. Subjects were instructed to draw accurately, to try to not write outside the lines, and to not lift up the stylus from the screen while drawing. The drawing task was performed three times after the subjects had practiced using the PC tablet and stylus and after they were accustomed to the necessary movements. Based on the position coordinates of the stylus on the PC tablet, the distance that the stylus strayed outside of the parallel lines (hereafter referred to as stray distance) and the speed of the stylus movement (hereafter referred to as speed) were calculated. 

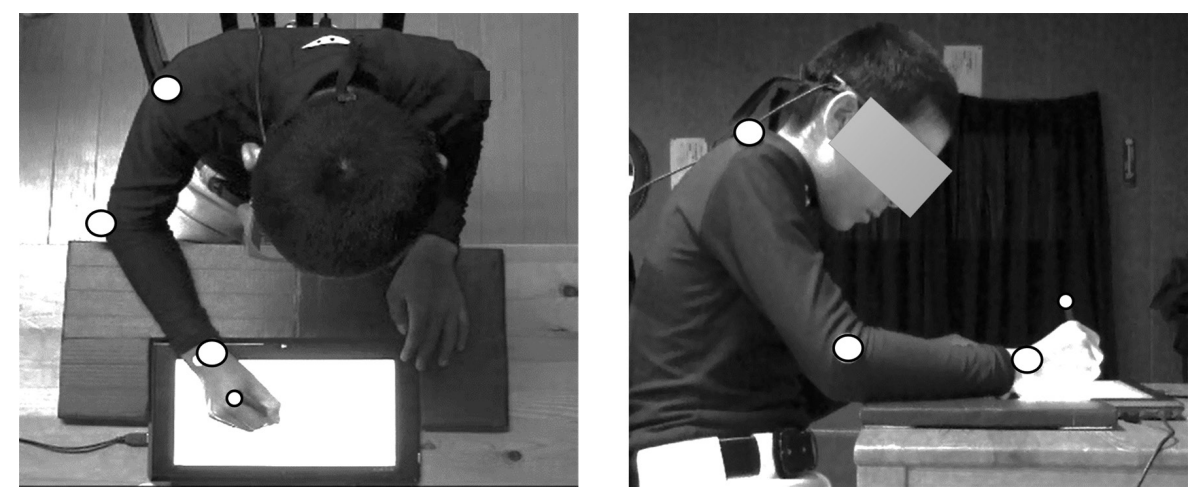

Fig. 1. Environment Configuration of Drawing Motion Analysis.

Note. Video cameras were placed above and to the right of the subjects to record the drawing motion, and markers were placed on the right acromion, the lateral epicondyle of the right humerus, right radial styloid process, and stylus to serve as indicators for the analysis of actions.

Dartfish 4.0 (Dartfish Company) was used to analyze the movements of each marker recorded by the cameras placed above and to the right of the subjects. The markers attached to the right acromion, lateral epicondyle of the right humerus, right radial styloid process, and stylus during the drawing tasks indicated movements of the body, shoulder joint, elbow joint, and fingers, respectively (Fig. 1). We calculated the trace length for each marker (hereafter referred to as sway length) and the total trace length (hereafter referred to as total sway length) while performing the drawing task. The ratio of the sway length for each marker was calculated by setting the total sway length of each marker to $100 \%$.

\section{Physical Function}

Twenty-six tasks were selected from the clinical observational assessment items of the Japanese Academy of Sensory Integration: three tasks were related to the postural reflex, three tasks were related to the residual primitive reflexes, four tasks were related to the muscle tone condition, two tasks were related to posture maintenance, four tasks were related to cooperativeness of the upper limbs, six tasks were related to compound motions and four tasks were related to eye movements. The tasks were conducted by occupational therapists in accordance with the procedures in the manual, and some subjects were filmed during the tests when the subjects and their parents gave consent.

The result of each task was classified into one of three categories: poor, moderately poor, or normal. Based on these results, the occupational therapist responsible for the experiment and a second occupational therapist with at least 5 years of clinical experience confirmed the scores while checking the recordings. In cases where subjects were not filmed, the second occu- pational therapist re-evaluated the clinical observations and, if the scores did not match, the two assessors discussed the results and confirmed the scores. The results of the clinical observations were quantified as follows: poor, 2 points; moderately poor, 1 point; and normal, 0 points. Total points for the seven categories and all tasks were then calculated.

\section{Data Analysis}

\section{Drawing Performance}

For healthy children, we calculated the largest stray distance in three times of the drawing task, the speed of the trial with the largest sway length, the difference between the fastest and the slowest speed (hereafter referred to as the range of speed) for each school grade, and the difference between the fastest and slowest speed of each healthy children (hereafter referred to as the difference of speed). For clumsy children, the largest stray distance, the range of speed, and the difference of speed were calculated and then compared with those of healthy children of the same grade. Clumsy children who had a larger stray distance than the largest stray distance of the healthy children of their grade were considered to have a drawing problem. Clumsy children who had a faster speed than the fastest speed of the healthy child group of each grade were defined as having a fast drawing speed. Similarly, those who had a slower speed than the slowest speed of the healthy child group of each grade were defined as having a slow drawing speed. Clumsy children who had a larger range of speed than the largest range of speed of the healthy child group of each grade were considered to have a varied drawing.

\section{Drawing Motion}

In regards to drawing motion in the healthy child 
group, we calculated the mean and standard deviation (SD) of both the sway length and the total sway length for each marker for each grade. Subjects who had a sway length of greater than the mean value $+1 \mathrm{SD}$ of the healthy child group were defined as having a large sway length, and those who had a sway length of less than the mean value $-1 \mathrm{SD}$ were defined as having a little sway length. We concluded that the clumsy children had different drawing motion than healthy children when their sway length was qualified as large or little.

Further, we calculated the mean ratio of the sway length for each marker for each grade. A goodness-offit test was used to analyze the ratio of sway length. The mean ratio value of the healthy child group for each grade was defined as the expected value and the mean ratio of the each clumsy child in each grade was defined as the actual measurements. When the ratio of sway length for each marker in the healthy child group and the clumsy child did not match, we concluded that the drawing motion differed between healthy child group and the clumsy child.

IBM SPSS statistical software version $16.0 \mathrm{~J}$ for Windows (IBM Global Services Japan Solution and Services Company) was used for statistical analysis and $p<0.05$ was used to determine statistical significance.

\section{Physical Function}

The physical function tasks were measured using the assessment scores of each area of the clinical observations and converted to a score with a possible total of 100 points. In the present study, subjects who had high assessment scores and high total points were determined as having a physical function problem.

Finally, clumsy children who had any problem in drawing performance or drawing motion were classified and analyzed according to trends noted in their physical dysfunction.

\section{Results}

\section{Drawing Performance}

The largest stray distance, the range of speed and the difference of speed for the healthy child group by grade and each clumsy child are shown in Table 1. Clumsy children were classified into two groups according to the largest stray distance: 6 children in Group I (Case A, B, G, H, I and J) had a drawing problem; 4 children in Group II (Case C, D, E and F) did not have a drawing problem. In clumsy children from Group I, they had a tendency for larger mean stray distance and greater speed variability than the healthy child group, when categorized by grade.

Table 1. Drawing performance for healthy and clumsy children.

\begin{tabular}{|c|c|c|c|c|c|c|}
\hline & \multirow{2}{*}{ Grade (age) } & \multicolumn{2}{|c|}{$\begin{array}{l}\text { the trial of the largest } \\
\text { stray distance }\end{array}$} & \multirow{2}{*}{ range of speed } & \multicolumn{2}{|c|}{ difference of speed } \\
\hline & & sway length & speed & & $\min$ & $\max$ \\
\hline \multirow{5}{*}{ Healthy children } & $2(7-8)$ & 2.39 & 2.13 & $1.36-3.28$ & 0.16 & 1.32 \\
\hline & $3(8-9)$ & 2.32 & 2.44 & $0.95-4.10$ & 0.24 & 1.97 \\
\hline & $4(9-10)$ & 1.80 & 2.69 & $0.96-3.11$ & 0.03 & 0.85 \\
\hline & $5(10-11)$ & 1.34 & 2.22 & $0.88-3.59$ & 0.14 & 1.37 \\
\hline & $6(11-12)$ & 0.20 & 1.74 & $1.20-2.67$ & 0.05 & 0.76 \\
\hline & \multirow[t]{2}{*}{ grade } & \multicolumn{2}{|c|}{$\begin{array}{l}\text { the trial of the largest } \\
\text { stray distance }\end{array}$} & \multirow[t]{2}{*}{ range of speed } & \multirow{2}{*}{\multicolumn{2}{|c|}{ difference of speed }} \\
\hline & & sway length & speed & & & \\
\hline \multirow{6}{*}{$\begin{array}{l}\text { Group I } \\
\text { with drawing problem }\end{array}$} & A (2) & $3.70 *$ & $4.79 *$ & $2.10-4.79$ & \multicolumn{2}{|c|}{$2.69 *$} \\
\hline & B (2) & $2.94 *$ & $2.15^{*}$ & $1.96-2.72$ & \multicolumn{2}{|c|}{0.76} \\
\hline & G (5) & $5.18^{*}$ & $2.65^{*}$ & $1.10-2.65$ & \multicolumn{2}{|c|}{$1.55^{*}$} \\
\hline & $\mathrm{H}(5)$ & $1.54 *$ & 1.30 & $0.91-2.63$ & \multicolumn{2}{|c|}{$1.72 *$} \\
\hline & $\mathrm{I}(6)$ & $1.58^{*}$ & $3.84 *$ & $2.48-3.84$ & \multicolumn{2}{|c|}{$1.36^{*}$} \\
\hline & $\mathrm{J}(6)$ & $13.76^{*}$ & $7.26^{*}$ & $3.93-7.26$ & \multicolumn{2}{|c|}{$3.33^{*}$} \\
\hline \multirow{4}{*}{$\begin{array}{l}\text { Group II } \\
\text { without drawing problem }\end{array}$} & C (3) & 0.27 & 2.43 & $1.95-2.81$ & \multicolumn{2}{|c|}{0.86} \\
\hline & D (3) & 1.94 & $3.07 *$ & $3.07-4.57$ & \multicolumn{2}{|c|}{1.50} \\
\hline & E (3) & 2.18 & 1.03 & $1.03-2.94$ & \multicolumn{2}{|c|}{1.91} \\
\hline & F (4) & 0.34 & 1.09 & $1.09-1.39$ & \multicolumn{2}{|c|}{0.30} \\
\hline
\end{tabular}

Stray distance; $\mathrm{cm}$, Speed; $\mathrm{cm} / \mathrm{sec}, *$; above the standard of the healthy child group of each grade Note. In clumsy children from Group I, they had a tendency for longer mean stray distance and greater speed variability than the healthy child group, when categorized by grade. 


\section{Drawing Motion}

The mean sway length and total sway length of each marker for the healthy child group and the clumsy children were classified into two groups according to drawing performance are shown in Table 2. The sway length and total sway length of each marker for clumsy children were defined as being large when the sway length deviated above the normal range of movement as measured by one of the cameras monitoring the subject. The sway length was defined as little than the normal range when the sway length deviated below the normal range as measured by either camera monitoring the subject.

We then compared the trends in the drawing motion between the two drawing performance groups are shown in Table 3. In clumsy children from Group I, most markers deviated from the normal range. In clumsy children from Group II, most markers remain within the normal range. Further, body sway length in all Group II subjects was within the normal range.

The goodness-of-fit test results for the ratio of sway length and total sway length for the healthy and clumsy children were classified into two groups are shown in Table 3. When the goodness-of-fit test revealed that the sway length ratio of the clumsy children differed from those of the healthy children group. And then there were differences in normal range of movement as measured by either camera monitoring the subject, the sway length ratio for each marker was determined not to be a fit.
Therefore, we compared the trends in drawing motion between the two groups from the results of the drawing performance tests. All children in Group I had differing ratios when compared with individuals in the healthy child group, and were therefore determined not to fit.

\section{Physical Function}

The clinical observations of drawing performance for each of the two groups of clumsy children are shown in Fig. 2. The results revealed numerous problems, including residual primitive reflexes, muscle tone condition, posture maintenance, cooperativeness of the upper limbs, and eye movement in both groups. However, a tendency for greater severity of problems was observed in Group I than that in Group II. Furthermore, a tendency for increased severity of problems related to postural reflex was noted in Group I. The total point score revealed that subjects in Group II tended to have fewer problems related to physical function characteristics.

\section{Discussion}

\section{Drawing Performance and Drawing Motion}

We found that some clumsy children who had poor drawing skill tended to have large motion of the body and shoulder joint for all drawing tasks, as compared to healthy children. On the other hand some clumsy children who tended to use finger movements to draw and exhibit a small handwriting motion, as compared

Table 2. The mean sway length and total sway length of each for healthy and clumsy children.

\begin{tabular}{|c|c|c|c|c|c|c|c|c|c|c|c|}
\hline & \multirow{2}{*}{$\begin{array}{c}\text { Grade } \\
\text { (age) }\end{array}$} & \multicolumn{5}{|c|}{ sway length (above) } & \multicolumn{5}{|c|}{ sway length (right side) } \\
\hline & & body & shoulder & elbow & finger & total & body & shoulder & elbow & finger & total \\
\hline \multirow{6}{*}{ Healthy children } & $3(8-9)$ & $1.01(0.63)$ & $0.74(0.44)$ & $1.10(0.63)$ & $2.09(0.87)$ & $4.91(1.92)$ & $0.90(0.57)$ & $0.91(0.53)$ & $0.57(0.45)$ & $1.05(0.71)$ & 3.39 (1.69) \\
\hline & $4(9-10)$ & $0.86(0.62)$ & $0.59(0.37)$ & $0.92(0.48)$ & $1.83(0.58)$ & $4.19(1.53)$ & $0.74(0.55)$ & $0.55(0.38)$ & $0.55(0.48)$ & $0.87(0.56)$ & $2.72(1.53)$ \\
\hline & $5(10-11)$ & $0.73(0.57)$ & $0.67(0.38)$ & $1.14(0.56)$ & $2.38(1.09)$ & $4.93(1.99)$ & $0.67(0.45)$ & $0.67(0.38)$ & $0.64(0.46)$ & $1.05(0.71)$ & $3.02(1.63)$ \\
\hline & $6(11-12)$ & $0.60(0.41)$ & $0.48(0.40)$ & $0.96(0.72)$ & $2.02(0.62)$ & $4.08(1.63)$ & $0.64(0.46)$ & $0.75(0.44)$ & $0.66(0.64)$ & $1.33(0.56)$ & $3.38(1.37)$ \\
\hline & \multirow{2}{*}{ grade } & \multicolumn{5}{|c|}{ trace length (above) } & \multicolumn{5}{|c|}{ trace length (right side) } \\
\hline & & body & shoulder & elbow & finger & total & body & shoulder & elbow & finger & total \\
\hline \multirow{4}{*}{$\begin{array}{l}\text { Group I } \\
\text { with drawing } \\
\text { problem }\end{array}$} & $\mathrm{A}(2)$ & 17.46 & 22.17 & 24.39 & 30.13 & 94.15 & 9.40 & 22.03 & 16.19 & 16.27 & 63.89 \\
\hline & B (2) & 28.73 & 28.64 & 36.44 & 37.76 & 131.57 & 20.04 & 30.02 & 16.43 & 19.99 & 86.49 \\
\hline & G (5) & 15.68 & 14.52 & 17.67 & 21.37 & 69.24 & 10.97 & 10.46 & 7.64 & 11.11 & 40.18 \\
\hline & $\mathrm{J}(6)$ & 4.01 & 3.15 & 14.43 & 19.05 & 40.65 & 2.61 & 4.93 & 9.59 & 10.74 & 27.86 \\
\hline \multirow{4}{*}{$\begin{array}{l}\text { Group II } \\
\text { without drawing } \\
\text { problem }\end{array}$} & C (3) & 15.56 & 22.66 & 36.18 & 34.83 & 109.23 & 6.47 & 19.37 & 11.67 & 14.26 & 51.78 \\
\hline & $\mathrm{D}(3)$ & 10.13 & 20.06 & 31.00 & 29.72 & 90.92 & 5.71 & 20.88 & 14.90 & 14.01 & 55.51 \\
\hline & E (3) & 8.05 & 10.72 & 18.97 & 23.64 & 61.38 & 3.43 & 5.88 & 11.18 & 12.19 & 32.67 \\
\hline & $\mathrm{F}(4)$ & 15.30 & 12.25 & 20.37 & 30.22 & 78.15 & 5.33 & 8.67 & 7.87 & 12.71 & 34.57 \\
\hline
\end{tabular}

Trace length : cm

Note. The mean sway length and total sway length of each marker for the healthy child group and the clumsy children were classified into two groups according to drawing performance. 
Table 3. Drawing motion by mean sway length and total sway length in clumsy children.

\begin{tabular}{|c|c|c|c|c|c|c|c|c|c|c|c|c|c|}
\hline & \multirow{3}{*}{$\begin{array}{l}\text { child } \\
\text { (grade) }\end{array}$} & \multicolumn{5}{|c|}{ sway length judgment } & \multicolumn{7}{|c|}{ goodness of fit test } \\
\hline & & \multirow{2}{*}{ body } & \multirow{2}{*}{ sholder } & \multirow{2}{*}{ elbow } & \multirow{2}{*}{ finger } & \multirow{2}{*}{ total } & \multicolumn{3}{|c|}{ above } & \multicolumn{3}{|c|}{ right side } & \multirow{2}{*}{ judgment } \\
\hline & & & & & & & $\mathrm{X}^{2}$ & df & $p$ value & $\mathrm{X}^{2}$ & df & $p$ value & \\
\hline \multirow{6}{*}{$\begin{array}{l}\text { Group I } \\
\text { with drawing } \\
\text { problem }\end{array}$} & $\mathrm{A}(2)$ & large & large & & & large & 7.7107 & 3 & $\geqq 0.05$ & 2.2623 & 3 & 0.52 & non fit \\
\hline & B (2) & large & large & & large & large & 13.1850 & 3 & $<0.01$ & 12.9190 & 3 & $<0.01$ & non fit \\
\hline & $\mathrm{G}(5)$ & large & & little & & & 14.7510 & 3 & $<0.01$ & 17.1680 & 3 & $<0.01$ & non fit \\
\hline & $\mathrm{H}(5)$ & large & large & & large & large & 44.7810 & 3 & $<0.01$ & 8.7063 & 3 & $<0.05$ & non fit \\
\hline & $\mathrm{I}(6)$ & little & little & little & little & little & 20.9390 & 3 & $<0.01$ & 34.0620 & 3 & $<0.01$ & non fit \\
\hline & $\mathrm{J}(6)$ & little & little & little & little & little & 10.7470 & 3 & $<0.05$ & 16.3830 & 3 & $<0.01$ & non fit \\
\hline \multirow{4}{*}{$\begin{array}{l}\text { Group II } \\
\text { without drawing } \\
\text { problem }\end{array}$} & $\mathrm{C}(3)$ & & large & & & large & 1.1378 & 3 & 0.77 & 1.4787 & 3 & 0.69 & \\
\hline & $\mathrm{D}(3)$ & & & & & & 0.6731 & 3 & 0.88 & 2.0445 & 3 & 0.56 & \\
\hline & $\mathrm{E}(3)$ & & little & & & little & 0.8870 & 3 & 0.83 & 11.6710 & 3 & $<0.01$ & non fit \\
\hline & F (4) & & little & little & & & 7.6320 & 3 & $\geqq 0.05$ & 4.4216 & 3 & 0.22 & non fit \\
\hline
\end{tabular}

Note. A goodness of fit test was used to analyze the ratio of sway length. The mean value of the healthy child group scores for each grade were defined as the expected values and the ratio of sway length of each clumsy child in each grade were defined as the actual measurements.

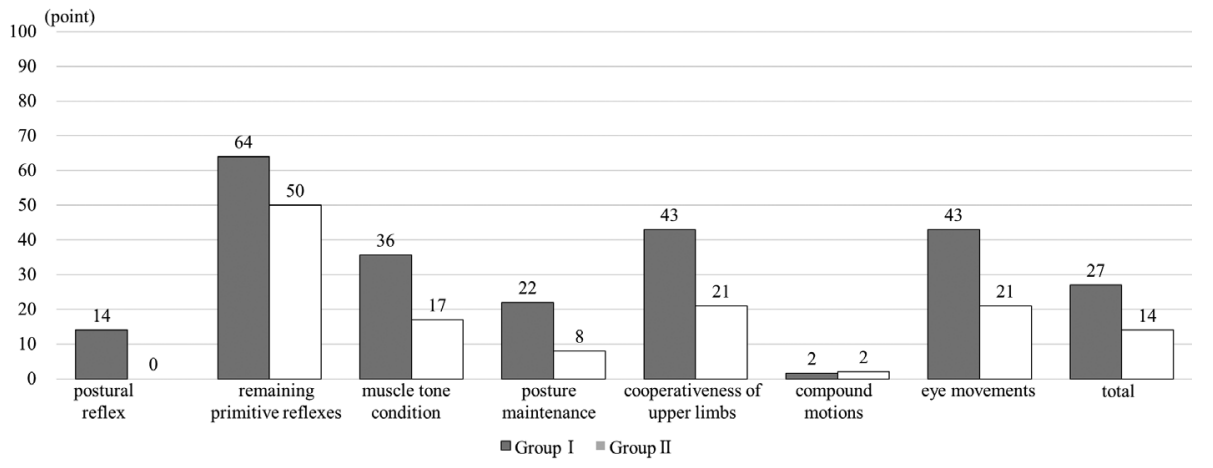

Fig. 2. The Physical Function in Clumsy Children.

Note. A tendency for increased severity of problems related to postural reflex was noted in Group I. The total point score revealed that subjects in Group II tended to have fewer problems related to physical function characteristics.

to healthy children. In either case, the drawings were inaccurate due to drawing problems, the mechanism by which these drawing problem manifest are not uniform.

The drawing tasks used in the present study contained an equilateral triangle with $10 \mathrm{~cm}$ sides. Tasks such as this that involve drawing large lines require movements of the shoulder and elbow joints $[11,12]$. Therefore, inaccurate drawings may be associated with instability of the body or scapula, or decreased cooperativeness of the upper limbs. When the drawing motion was generally large, movements of the body and shoulder joints also tended to become larger due to posture instability. This is similar to the results of previous studies [13] and may be due to the influence of problems in maintaining continued muscle tone [7]. Children who have decreased muscle tone and problems in maintaining continued muscle tension often stabilize or immobilize another part of the body for increased support [14]. Subjects who used small drawing motions to draw the $10 \mathrm{~cm}$ triangle placed their upper limbs on the desk to obtain support for handwriting, allowing them to compensate for the instability in posture and problems in maintaining continued muscle tension.

\section{Physical Function}

The results of the clinical observations revealed that when drawings were inaccurate, there were problems with postural reflexes, posture maintenance, or cooperativeness of the upper limbs. However, based on the results of the clinical observations, the trends observed for characteristics of physical function problems in clumsy children whose drawings were inaccurate appeared to be similar, but the severity of the problems was unclear.

Each clumsy child used a different strategy when using the writing tools to cope with their individual deficits in physical function, which may have led to differences in drawing motion. However, the strategies used by clumsy children did not seem to be appropriate, 
and the subjects could not control the writing tools accurately.

Moreover, body stability appeared to have the most influence on drawing accuracy in clumsy children who had no problems in drawing, and had similar drawing motion as healthy children. This was based on the fact that these subjects tended to show no problems in posture reflexes, posture maintenance, or performance of composite movements.

\section{Study Limitation}

It may be inappropriate to apply the results of this research to females, because this research was performed exclusively in male subjects. The figure in this drawing task was a triangle; hence, these results may not apply directly to other drawing tasks. A problem of handwriting indicated by daily life is that it includes writing as well as drawing. The drawing task performed in this study can generate a quantitative result, but handwriting evaluation is inevitably subjective to some degree. We can't evaluate three-dimensional movement enough because this study analyzed drawing motion of two dimensions movement analysis software.

\section{Implication for Occupational Therapy Practice}

The results of our study have the following implications for occupational therapy practice:

- The evaluation of physical function is necessary to a supported occasion for a problem of drawing, and we have to confirm the muscle tone condition, posture maintenance, cooperativeness of the upper limbs and eye movement in particular

- Children with drawing problems exhibit different drawing motion than healthy children, and it is necessary to support it in drawing motion as well as the physical function.

\section{Conclusion}

From our findings, clumsy children with developmental disorders seem to present with difficulty in physical functions. Limitations in drawing and drawing motion are influenced primarily by body stability. Moreover, although we originally thought that the severity of problems in muscle tone and cooperativeness of the upper limbs had an influence on the ability to control writing tools, in some cases, subjects could nevertheless control the tools accurately depending on the method of drawing.

When providing support to children who have problems in writing or drawing, improvements may be gained by teaching them the strategies that are uniquely used by healthy children. However, it is possible that changing strategies may decrease drawing ability in clumsy children. In order to examine methods of support for the physical function of individual clumsy children, it is necessary to not only evaluate the drawing performance and physical function of subjects but also to assess the methods of drawing.

Acknowledgements: This study received a grant from Sapporo Medical University, Japan. This study was a part of the first author's thesis for a doctorate.

\section{References}

[1] Casellato C, Zorzi G, Pedrocchi A, Ferrigno G, and Nardocci N. Reaching and writing movements: sensitive and reliable tools to measure genetic dystonia in children. J Child Neurol. 2011; 26(7): 822-9.

[2] Nakajima S, Ohyanagi T, Nakamura Y, Sakamoto K, and Sengoku Y. The assessment of handwriting performance based on changes in pen velocity and pen pressure (in Japanese). The Journal of Japanese Occupational Therapy research (sagyouryouhou). 2011; 30(5): 563-71.

[3] Nakajima S, Ohyanagi T, Nakamura Y, Takizawa S, Ikeda C, and Sengoku Y. Developmental characteristics of handwriting performance of normal children and healthy adults (in Japanese). Japan Journal of Occupational Therapy in Pediatrice (nihonhattatukeisagyouryouhougakkaishi). 2014; 3(1): 46-52.

[4] Sakamoto K, Nakajima S, Sera A, Ohyanagi T, and Sengoku Y. Relationship between drawing line performance and other abilities of clumsy children with developmental disorder (in Japanese). Japan Journal of Occupational Therapy in Pediatrice (nihonhattatukeisagyouryouhougakkaishi). 2012; 1(1): 39-45.

[5] Olive T, and Kellogg RT. Concurrent activation of highand low-level production processes in written composition. Mem Cognit. 2002; 30(4): 594-600.

[6] Van Hoorn JF, Maathuis CG, Peters LH, and HaddersAlgra M. Handwriting, visuomotor integration, and neurological condition at school age. Dev Med Child Neurol. 2010; 152(10): 942-7.

[7] Chang SH, and Yu NY. Characterization of motor control in handwriting difficulties in children with or without developmental coordination disorder. Dev Med Child Neurol. 2010; 52(3): 244-50.

[8] Klein S, Guiltner V, Sollereder P, et al. Relationships Between Fine-Motor, Visual-Motor, and Visual Pereseption Scores and Handwriting Legibility and Speed. Phys Occup Ther Pediatr. 2011; 31(1): 103-14.

[9] Giroux P, Woodall W, Weber M, and Bailey J. Occupational Therapy Practitioners' Perceptions of Important Competencies for Handwriting Evaluation and Intervention in School-Aged Children. Phys Occup Ther Pediatr. 2012; 32(1): 66-79.

[10] Ohyanagi T, Nakajima S, Nakamura Y, and Sengoku Y. Development of new software for the assessment of upper 
extremity function with handwriting task (in Japanese). Sapporo Medical University bulletin of School of Health Sciences (sapporoikadaigakuhokenniryougakubukiyou). 2010; $12: 1-8$.

[11] Lacquanti F, Ferrigno G, Pedotti A, Soechting JF, and Terzuolo C. Changes in spatial scale in drawing and handwriting. J Neurosci. 1987; 7(3): 819-28.

[12] Van Drempt N, McCluskey A, and Lannin NA. A review of factors that influence adult handwriting performance.
Aust Occup Ther J. 2011; 58(5): 321-8.

[13] Flatters I, Mushtaq F, Hill LJ, Holt RJ, wilkie RM, and Mon-Williams M. The relationship between a child's postural stability and manual dexterity. Exp Brain Res. 2014; 232(9): 2907-17.

[14] Nichols DS. Development of postural control. In J CaseSmith, editor. Occupational therapy for children, 4th ed. St Louis, Mosby; 2001; 266-88. 